\section{Third Cerebellar Hemisphere: An Unusual New Cerebellar Anomaly}

Improved visualization of the posterior fossa structures has lead to an increased recognition of cerebellar malformations, including the Dandy-Walker malformation, Joubert syndrome, rhombencephalosynapsis, tectocerebellar dysrhaphia, and so forth. New anomalies continue to be discovered, highlighting the fact that cerebellar anomalies are poorly understood and have largely been ignored in the literature. We present a structural anomaly of the cerebellum, which we believe has not been previously reported.

A 16-month-old girl presented to the pediatric outpatient department with some delayed developmental milestones. She was full-term with a normal vaginal delivery and no history suggestive of perinatal asphyxia. The motor milestones were delayed, and the child could not stand. The other milestones, including language and socialization, were normal. Examination revealed a bony hard swelling in the occipital region, which, according to the mother, was noticed soon after birth. The occipitofrontal circumference was $52 \mathrm{~cm}$, and the anterior fontanelle was open. There was generalized hypotonia, and the deep tendon reflexes were depressed. Mild truncal ataxia was observed, but there was no nystagmus.

On CT examination, she was found to have a bony outpouching with a small defect in the bone outline on the left side. Within this outpouching, there was a third cerebellar hemisphere, which appeared to be only mildly dysplastic, and it was attached to the medulla on the right side by a long thin band of soft tissue of attenuation similar to that of the cortical gray matter. MR imaging confirmed the findings of CT (Figs 1-3). However, there was evidence of basilar invagination in addition and low-lying torcular herophilli. The vermis was formed only superiorly, and the supratentorial parenchyma was normal.

In this index case, the vermis was formed only superiorly, and there was a mildly dysplastic third cerebellar hemisphere. This anomaly, to the best of our knowledge, had not been reported earlier.

The understanding of a cerebellar malformation is facilitated by knowledge of the process of early fetal cerebellar development. During the fifth week of gestation, thickening occurs bilaterally in the alar plate of the rhombencephalon, forming the rhombic lips, which are the primordia of the cerebellar hemispheres. ${ }^{1,2}$ The neuroepithelial zones, in the roof of the fourth ventricle and the rhombic lips, are the location of germinal matrices, where the cells of the cerebellum and many brain stem nuclei will form. ${ }^{3}$ The cerebellar vermis is formed on fusion of the developing hemispheres when they meet superiorly at the midline during the ninth gestational week; fusion then continues inferiorly as the hemispheres grow. ${ }^{1}$

The possible pathophysiology leading to malformation in this index case could be mutation of a gene expressed in cerebellar development, leading to development of an extra rhombic lip. Pre- and perinatal injury is less likely because the remainder of the supratentorial and infratentorial structures did not reveal any abnormality.

Jackson et $\mathrm{al}^{4}$ reported a case of unilateral duplication of the cerebellar hemisphere and petrous bone, with associated structures. Developmentally, the anomalous structures were derived from diverse embryologic tissues, and the authors traced the origin of the defect to the third week of embryonic development. These findings are very different from the findings seen in our patient.

The purpose of this case report was to present an unusual cerebellar malformation, which we believe has not been reported so far.

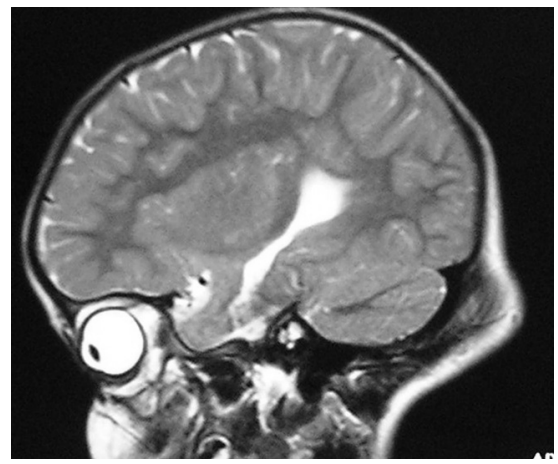

Fig 1. Sagittal T2-weighted MR image reveals a normal left cerebellar hemisphere. The visualized supratentorial parenchyma appears normal.

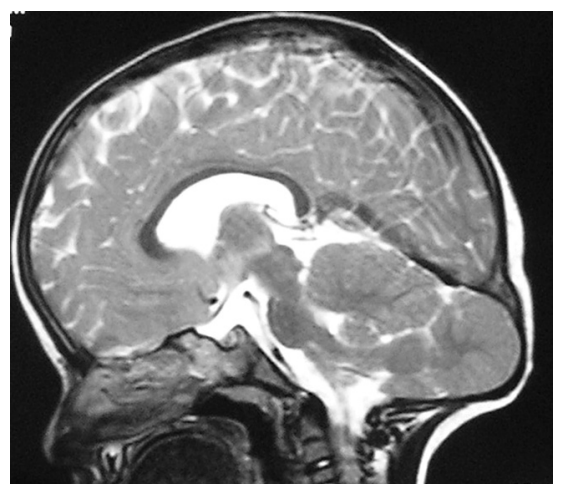

Fig 2. Sagittal T2-weighted MR image reveals a mildly dysplastic third cerebellar hemisphere located within a bony outpouching connected to the medulla, with a thin band of soft tissue of signal intensity similar to that of the cortex. In addition, it also reveals a low-lying torcular herophilli and mild basilar invagination. The visualized supratentorial parenchyma appears normal.

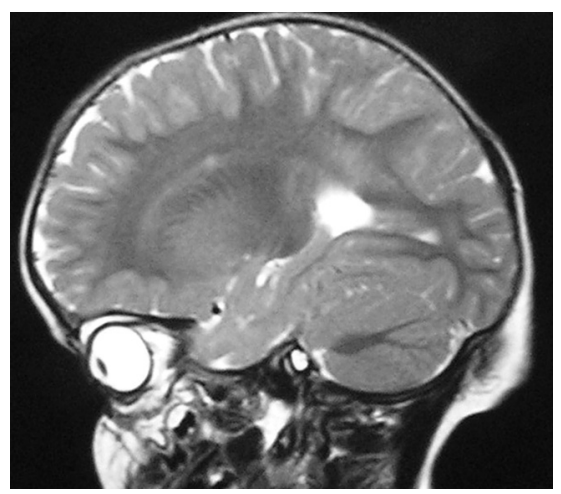

Fig 3. Sagittal T2-weighted MR image reveals a normal right cerebellar hemisphere The visualized supratentorial parenchyma appears normal.

S. Agarwal

Department of Radiodiagnosis

G. Gathwala

Department of Pediatrics

Post Graduate Institute of Medical Sciences

Rohtak, India

\section{References}

1. Barkovich AJ. Pediatric Neuroimaging. 2nd ed. New York: Raven Press; 1995:246-57

2. Freide RL. Developmental Neuropathology. 2nd ed. Berlin, Germany: SpringerVerlag; 1989:361-71 
3. Lin JC, Cai L, Cepko CL. The external granular layer of the developing chick cerebellum generates granule cells and cells of the isthmus and rostral hindbrain. J Neurosci 2001;21:159-68

4. Jackson JM, Sadove AM, Weaver DD, et al. Unilateral duplication of the cere- bellar hemisphere and internal, middle, and external ear: a clinical case study. Plast Reconstr Surg 1990;86:550-53

DOI 10.3174/ajnr.A2473 